\title{
Use of angle kappa in myopic photorefractive keratectomy
}

\author{
This article was published in the following Dove Press journal: \\ Clinical Ophthalmology \\ 29 January 2015 \\ Number of times this article has been viewed
}

\author{
Hamid Khakshoor' \\ Michael V McCaughey ${ }^{2}$ \\ Amir Hossein Vejdani' \\ Ramin Daneshvar' \\ Majid Moshirfar ${ }^{3}$ \\ 'Department of Ophthalmology, \\ The University of Mashhad, Mashhad, \\ Iran; ${ }^{2}$ Department of Ophthalmology, \\ The University of New Mexico, \\ School of Medicine, Albuquerque, NM, \\ USA; ${ }^{3}$ Department of Ophthalmology, \\ Francis I Proctor Foundation, \\ University of California San Francisco, \\ CA, USA
}

Purpose: To explore utilization of the coaxially sighted corneal light reflex (CSCLR) for centration during myopic photorefractive keratectomy (PRK) for patients with relatively high angle kappa ( $\kappa)$ values.

Methods: Patients were stratified into two groups preoperatively, on the basis of angle $\kappa$ values. Group A was composed of 166 eyes with an angle $\kappa$ value $<5^{\circ}$. Group B consisted of 182 eyes with an angle $\kappa$ value $>5^{\circ}$. Intraoperative centering of ablation was performed within group $A$ by utilizing the pupillary center, and within group B by using the CSCLR. Visual acuities were evaluated and compared at 6 months and 12 months postoperatively between groups.

Results: Mean uncorrected visual acuities (UCVA) for all patients at 6 months and 12 months were $-0.073 \log$ MAR and $-0.080 \log$ MAR, respectively. A total of $98.9 \%$ of patients had a UCVA of $0.00 \operatorname{logMAR}(\approx 20 / 20$ Snellen $) 12$ months postoperatively. There was not a significant between-group difference in regard to residual refractive error at 6 months or 12 months ( $P=0.53$ and $P=0.97)$, or in UCVA at 6 months and 12 months $(P=0.76$ and $P=0.17)$. There were no subjective complaints of monocular diplopia, glare, or haloes within either group at any time during follow-up.

Conclusion: Availing use of the CSCLR for centration of ablation within myopic patients with high angle $\kappa$ values may aid in providing better refractive outcomes after performance of PRK.

Keywords: angle kappa, photorefractive keratectomy, PRK, CSCLR, myopia, Purkinje reflex

\section{Introduction}

We would like to share our preliminary report concerning the utilization of angle kappa ( $\kappa)$ during myopic photorefractive keratectomy (PRK) for reducing the incidence of postoperative visual complaints. Angle $\kappa$ is a measure of the discrepancy between the pupillary axis and visual axis. Using the pupillary axis for determination of centration during refractive surgery can result in misalignment of ablation when angle $\kappa$ is significantly positive $\left(>5^{\circ}\right)$. Misalignment of ablation can result in postoperative glare, induction of irregular astigmatic error, and diminuition of best spectacle corrected visual acuity (BSCVA). ${ }^{1,2}$ Resultantly, attainment of accurate ablation zone centration designates an important objective. Although many excimer laser platforms contain eye-tracking systems for the prevention of such errors, ${ }^{3-7}$ large discrepancies between the pupillary and visual axis within certain patients may still result in imprecise alignment of ablation. While a large angle $\kappa$ is more commonly associated with hyperopia, a study by Basmak et $\mathrm{al}^{8}$ found a positive angle $\kappa$ in $75.8 \%$ of myopes. Further, Hashemi et $\mathrm{al}^{9}$ report that the mean angle $\kappa$ in myopes is $5.13^{\circ} \pm 1.5^{\circ}$. Accounting for angle $\kappa$ through utilization of the coaxially sighted corneal light reflex (CSCLR) during hyperopic refractive surgery appears to represent a more preferable method
Correspondence: Majid Moshirfar Department of Ophthalmology, Francis I. Proctor Foundation, University of California San Francisco, 10 Koret Way, K-30I

San Francisco, CA 94I43, USA

$\mathrm{Tel}+\mathrm{I} 4155142800$

$\mathrm{Fax}+\mathrm{I} 4155146845$

Email majid.moshirfar@ucsf.edu 
for ensuring accuracy of centration intraoperatively. ${ }^{10}$ The synonymously used terms "CSCLR" and "Purkinje reflex" refer to the approximate supposed location of the visual axis; this visual representation therefore denotes a reference point for discerning the center of planned ablation. A study by Chan and Boxer Wachler ${ }^{11}$ found that mean decentration in hyperopes was reduced from $0.40 \pm 0.24 \mathrm{~mm}$ when angle $\kappa$ was ignored to $0.06 \pm 0.18 \mathrm{~mm}$ when angle $\kappa$ was accounted for. Our investigational objective was to comparatively analyze postoperative outcomes between two dissimilar groups in which differing techniques of ablative centration were performed.

\section{Materials and methods}

Records of patients who had PRK performed by a single surgeon were reviewed. All operations were done between 2006 and 2008 using a Bausch \& Lomb Technolas-217z (Bausch \& Lomb Surgical, Inc.) system. The study protocol was approved by the Ethical Committee of Mashhad University of Medical Sciences, Mashhad, Iran, and adhered to the tenets of the Declaration of Helsinki. Before surgery, a full ophthalmic examination was performed for all patients, including BSCVA, slit-lamp biomicroscopy, Goldmann applanation tonometry, full cycloplegic refraction, manifest refraction, funduscopic examination, and topographic and aberrometric evaluations with the Orbscan II (Bausch \& Lomb Surgical) and Zywave aberrometer (Bausch \& Lomb), respectively. Regular postoperative examinations were performed following surgery, and results during the 6th month and 12th postoperative months were considered for final statistical analysis. Preoperative angle $\kappa$ was evaluated and patients were grouped by angle $\kappa$. Group A comprised 166 eyes with an angle $\kappa<5^{\circ}$, and group B contained 182 eyes with an angle $\kappa>5^{\circ}$. During the procedure, group A patients, lying in the supine position with head in primary position, were asked to visually fixate on a blinking red target within the device aperture; the procedure was then performed according to the device protocol. Intraprocedurally, patients in group B were also asked to fixate on the red target, which resulted in an observed moderate outward deviation of the eye. The patient was asked to maintain fixation, and the surgeon manually adjusted the patient's head to center the Purkinje reflex (CSCLR) of the blinking target on the center of the patient's cornea; PRK was then performed in this new orientation using an identical procedural approach to that which was performed in group A. During each scheduled follow-up, a comprehensive ophthalmological examination was performed, and patients were questioned about occurrence of glare, haloes, and diplopia during all follow-up visits. A single trained ophthalmic technician unaware of group assignment performed all follow-up examinations.

Statistical analysis was performed using SPSS version 11 (SPSS Inc., Chicago, IL, USA). Obtained data were considered statistically significant with an obtained $P$-value of $<0.05$. Pearson's correlation was used to determine correlations between variables. Mean uncorrected visual acuities (UCVA) at 6 months and 12 months were compared between each group using the Student's $t$-test for independent samples.

\section{Results}

This study included a total of 348 eyes of 175 patients. There was a total of 123 females (35\%) and 225 males (65\%), with a mean age of $29.03 \pm 9.11$ years (range of 19-57 years). Group A contained a total of 166 eyes, and group B contained 182 . The two groups did not have any statistically significant difference in preoperative BSCVA or mean age. Mean UCVA for all patients at 6 months and 12 months was $-0.073 \log$ MAR and $-0.080 \log$ MAR, respectively, and $98.9 \%$ of all patients had a UCVA of $0.00 \log$ MAR (Snellen equivalent $=20 / 20$ ) at 12 months; there were no cases of UCVA line loss within either group. Refractive changes after 6 months and 12 months were not related to age (6 months: $P=0.88, r=0.009$ ) (12 months: $P=0.19, r=-0.078)$. Angle $\kappa$ did not correlate with age, but there was a high correlation with preoperative spherical equivalent, such that eyes with the highest angle $\kappa$ values also had the highest spherical equivalent values $(P<0.05, r=0.274)$. There was no significant difference between groups in relation to residual refractive error at 6 months or 12 months $(P=0.53$ and $P=0.97)$ or in UCVA at 6 months and 12 months $(P=0.76$ and $P=0.17)$. Importantly, there were no reports of monocular diplopia, glare, or haloes within either group at 12 months.

\section{Discussion}

Pupillary centering represents the predominant method for determination of proper intraoperative orientation, particularly within myopic patients. Patients possessing large angle $\kappa$ values can appear mildly exotropic while fixating on intraoperative indicators during initial alignment determination, with a resultant higher likelihood of sustaining a nasally decentered ablation. Compensation through lateral rotation of the patient's head aids in the prevention of this complication, through superimposition of the CSCLR and pupillary center. It has been previously recommended that pupillary center-based ablations result in greater accuracy 
of centration when compared with vertex-based ablations. ${ }^{12}$ A technique using a reference point midway between the pupillary center and CSCLR for patients with angle $\kappa$ values $>0.2 \mathrm{~mm}$ has also been utilized. ${ }^{13}$ Within certain patients possessing moderately high angle $\kappa$ values, pupillary based centering may be a desirable method, provided that the ablative diameter sufficiently encompasses the degree of visual/pupillary axis offset. While more research is indicated, we believe that by accounting for angle $\kappa$, the traditional complications associated with PRK in patients with a large angle $\kappa$ were prevented. We recommend that all myopic patients be preoperatively evaluated for angle $\kappa$, and that any patient with an angle $\kappa>5^{\circ}$ receive procedural alterations similar to the technique outlined here.

\section{Disclosure}

The authors report no conflicts of interest in this work.

\section{References}

1. Terrell J, Bechara SJ, Nesburn A, Waring GO, Macy J, Maloney RK. The effect of globe fixation on ablation zone centration in photorefractive keratectomy. Am J Ophthalmol. 1995;119(5):612-619.

2. Pande M, Hillman JS. Optical zone centration in keratorefractive surgery. Entrance pupil center, visual axis, coaxially sighted corneal reflex, or geometric corneal center? Ophthalmology. 1993;100(8):1230-1212.
3. Krueger RR. In perspective: eye tracking and autonomous laser radar. J Refract Surg. 1999;15(2):145-149.

4. Gobbi PG, Carones F, Brancato R, et al. Automatic eye tracker for excimer laser photorefractive keratectomy. J Refract Surg. 1995; 11(3 Suppl):S337-S342.

5. Tsai YY, Lin JM. Ablation centration after active eye-tracker-assisted photorefractive keratectomy and laser in situ keratomileusis. J Cataract Refract Surg. 2000;26:28-34.

6. Taylor NM, Eikelboom RH, van Sarloos PP, Reid PG. Determining the accuracy of an eye tracking system for laser refractive surgery. $J$ Refract Surg. 2000;16:S643-S646.

7. Mrochen M, Eldine MS, Kaemmerer M, Seiler T, Hütz W. Improvement in photorefractive corneal laser surgery results using an active eye-tracking system. J Cataract Refract Surg. 2001;27:1000-10065.

8. Basmak H, Sahin A, Yildirim N, Papakostas TD, Kanellopoulos AJ. Measurement of angle kappa with synoptophore and Orbscan II in a normal population. J Refract Surg. 2007;23:456-460.

9. Hashemi H, Khabaz Khoob M, Yazdani K, Mehravaran S, JafarzadehpurE, Fotouhi A. Distribution of angle kappa measurements with Orbscan II in a population-based survey. $J$ Refract Surg. 2010;26:966-971.

10. Reinstein DZ, Gobbe M, Archer TJ. Coaxially sighted corneal light reflex versus entrance pupil center centration of moderate to high hyperopic corneal ablations in eyes with small and large angle kappa. J Refract Surg. 2013;29(8):518-525.

11. Chan CC, Boxer Wachler BS. Centration analysis of ablation over the coaxial corneal light reflex for hyperopic LASIK. J Refract Surg. 2006;22:467-471.

12. Cavanaugh TB, Durrie DS, Riedel SM, Hunkeler JD, Lesher MP. Centration of excimer laser photorefractive keratectomy relative to the pupil. J Cataract Refract Surg. 1993;19 Suppl:S144-S148.

13. Lin DT, Sutton HF, Berman M. Corneal topography following excimer photorefractive keratectomy for myopia. J Cataract Refract Surg. 1993;19 Suppl:149-154.
Clinical Ophthalmology

\section{Publish your work in this journal}

Clinical Ophthalmology is an international, peer-reviewed journal covering all subspecialties within ophthalmology. Key topics include: Optometry; Visual science; Pharmacology and drug therapy in eye diseases; Basic Sciences; Primary and Secondary eye care; Patient Safety and Quality of Care Improvements. This journal is indexed on

Submit your manuscript here: http://www.dovepress.com/clinical-ophthalmology-journal

\section{Dovepress}

PubMed Central and CAS, and is the official journal of The Society of Clinical Ophthalmology (SCO). The manuscript management system is completely online and includes a very quick and fair peer-review system, which is all easy to use. Visit http://www.dovepress.com/ testimonials.php to read real quotes from published authors. 